\title{
SIMULAÇÃO DAS PERDAS DE ÁCIDO ASCÓRBICO DURANTE A PASTEURIZAÇÃO DO SUCO DE LARANJA
}

\author{
K. T. M. LIMA, S. D. V. TERRA e R. P. VIEIRA \\ Instituto Federal do Sul de Minas Gerais, Departamento de Engenharia Química \\ E-mail para contato: ronierik.vieira@ifsuldeminas.edu.br
}

\begin{abstract}
RESUMO - Simulações computacionais foram utilizadas para analisar a influência da temperatura e do tempo de pasteurização do suco de laranja do tipo "pêra" sobre a degradação de ácido ascórbico (vitamina C). Para isto, utilizou-se um modelo cinético previamente desenvolvido e validado. Os resultados mostraram que tanto a temperatura quanto o tempo de residência apresentam influências sobre a degradação desta substância. Entretanto, verificou-se que devido ao baixo valor da energia de ativação para a espécie considerada, a variação da temperatura apresentou pouca influência em pequenos tempos de residência. Assim, sugere-se a operação do sistema em temperaturas relativamente altas com um baixo tempo de residência.
\end{abstract}

\section{INTRODUÇÃO}

No Brasil, o suco concentrado da laranja é muito importante na economia, principalmente no estado de São Paulo, em que o capital gira em torno de US\$ 1 bilhão/ano. A partir dos anos 60 a citricultura brasileira voltou-se para a produção de matéria prima visando às necessidades indústrias e, desse modo, à preocupação com as frutas de mesa diminuiu, ocasionado barreiras não tarifárias nas exportações. Esse dado revela uma baixa participação do comércio internacional da laranja como fruta fresca. Entretanto o suco concentrado representa mais de $85 \%$ do capital envolvido nas exportações, isso mostra uma grande importância agroindustrial e da fruticultura nacional no comércio exterior (Neves et al., 2001). Dentre os fatores que fazem com que esta fruta represente uma grande parcela da produção de sucos no mudo, estão as características nutricionais como, por exemplo, os elevados níveis de vitamina $\mathrm{C}$.

O ácido ascórbico (AA) ou vitamina C, encontrada na laranja, é de suma importância para o organismo humano, sendo capaz de inibir a formação de metabólitos nitrosos carcinogênicos e, em ensaios biológicos, foi mostrada a grande capacidade de proteção que ela remete aos seres humanos, principalmente contra a exposição às radiações e aos medicamentos (Bianchi e Antunes, 1999). Além disso, a ingestão diária dessa vitamina se torna requerida e necessária pelo fato de o organismo humano ser incapaz de sintetizar o ácido ascórbico (Manela-Azulayi et al., 2003). Deste modo, torna-se primordial para a competitividade das indústrias produtoras de sucos de laranja, a alta qualidade do produto que, neste caso, está associada às elevadas concentrações de AA. 
Durante o processo de pasteurização realizado nas indústrias, que objetiva inibir enzimas e o crescimentos de microrganismos, ocorrem perdas do ácido ascórbico, sendo necessário um dimensionamento adequado à matéria prima que será utilizada de modo que essas perdas sejam mínimas. Os principais causadores da degradação do AA são a temperatura, tempo de contato, níveis de oxigênio, $\mathrm{pH}$ e outras características intrínsecas da fruta. Neste contexto, o presente trabalho fornece uma análise de alguns parâmetros reacionais (temperatura e tempo de residência) sobre as perdas de AA num sistema de troca térmica tubular. $\mathrm{O}$ objetivo é simular o processo utilizando uma modelagem cinética previamente desenvolvida e validada experimentalmente e, a partir da análise da influência dos parâmetros, propor direcionamentos para a redução das perdas desta importante substância.

\section{MODELAGEM MATEMÁTICA}

\subsection{Equação de taxa}

Assim como no trabalho de Burdurlu et al. (2006), um modelo cinético de primeira ordem foi utilizado para representar a taxa de degradação de ácido ascórbico em relação ao tempo (Equação 1), que pode ser facilmente integrada, obtendo-se a Equação 2, para um sistema em batelada.

$$
\begin{gathered}
\frac{d C_{A}}{d t}=-k C_{A} \\
C_{A}=C_{A 0} e^{-k t}
\end{gathered}
$$

em que $C_{A}$ representa a concentração de $\mathrm{AA}\left(\mathrm{mg} \mathrm{L}^{-1}\right)$ no tempo $t, C_{A 0}$ é a concentração inicial de AA e $k$ é a constante cinética de degradação do composto $\left(\mathrm{h}^{-1}\right)$, que depende da temperatura de acordo com a equação de Arrhenius (Equação 3).

$$
k=k_{0} e^{-E a / R T}
$$

em que $k_{0}$ é o fator pré-exponencial $\left(\mathrm{h}^{-1}\right), E_{a}$ é a energia de ativação $\left(\mathrm{J} \mathrm{mol}^{-1}\right)$ e $R$ a constante dos gases $\left(8,314 \mathrm{~J} \mathrm{~mol}^{-1} \mathrm{~K}^{-1}\right)$.

\subsection{Equacionamento para representar o sistema de pasteurização}

Neste trabalho, um sistema de pasteurização tubular, com capacidade de $0,1 \mathrm{~m}^{3}$, foi considerado como sistema. Diferentemente da abordagem de dimensionamento da capacidade de troca térmica, o pasteurizador foi considerado como um reator tubular, em que a degradação do ácido ascórbico foi simulada ao longo do sistema. A Figura 1 ilustra o sistema típico de pasteurizador duplo tubo. 
Figura 1 - Representação esquemática de um sistema de pasteurização tubular que será considerado como reator.

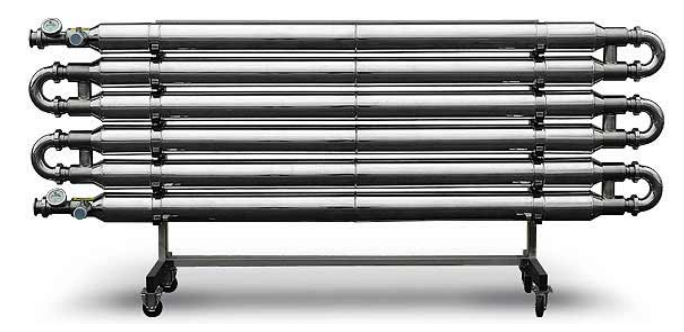

A fim de simplificar o modelo, o sistema da Figura 1 foi considerado como um reator tubular com fluido em escoamento uniforme, isto é, nenhum gradiente radial de concentração, temperatura ou de velocidade de reação foi considerado. À medida que o suco fresco (com concentração inicial de AA) escoa axialmente, no sentido da saída, a degradação do ácido se processa ao logo do comprimento do reator, sendo que a temperatura e o tempo de residência influenciarão consideravelmente no processo e serão analisadas por simulação. Sabe-se que a equação do balanço molar para a espécie A (neste caso o ácido ascórbico) em um reator tubular pode ser resolvida para obter o volume em função da conversão através da Equação 4.

$$
V=F_{A 0} \int_{0}^{X} \frac{d X}{-r_{A}}
$$

Substituindo a equação de taxa (Equação 1), expressa em termos de conversão, na Equação 4, obtêm-se a Equação 5, que é a equação de desempenho para o sistema de pasteurização considerado como reator tubular.

$$
X_{A}=1-e^{-k \tau}
$$

em que $X_{A}$ representa a perda de ácido ascórbico e $\tau$ o tempo espacial no sistema;

\subsection{Simulação e análise}

Para a condução das simulações, foi realizada uma validação prévia do modelo cinético com os dados experimentais obtidos do nosso grupo de pesquisa. Os detalhes da validação do modelo, bem como a determinação dos parâmetros cinéticos encontram-se no trabalho de Silva et al. (2015). No entanto, a fim de ilustrar a reprodutibilidade do modelo cinético obtido, uma comparação dos dados experimentais de degradação do ácido ascórbico com as simulações será fornecida na discussão dos resultados. A Tabela 1 fornece os parâmetros cinéticos que serão utilizados para analisar o processo por simulações.

Tabela 1 - Parâmetros determinados no trabalho de Silva et al. (2015) que serão utilizados nas simulações do presente trabalho.

\begin{tabular}{cc}
\hline Parâmetro & Valor \\
\hline Energia de ativação, $E a$ & $21 \mathrm{~kJ} \mathrm{~mol}^{-1}$ \\
Fator pré-exponencial, $k_{0}$ & $2208 \mathrm{~h}^{-1}$ \\
\hline
\end{tabular}

Foram influências 
do tempo de residência $(1,10$ e $20 \mathrm{~s})$ e, também, a influência da temperatura de pasteurização $\left(70,80\right.$ e $\left.90{ }^{\circ} \mathrm{C}\right)$ sobre a degradação do ácido ascórbico proveniente do suco de laranja com as características cinéticas expressas na Tabela 1.

\section{RESULTADOS E DISCUSSÃO}

A Figura 2 fornece uma comparação entre os dados experimentais previamente determinados pelo nosso grupo de pesquisa e as simulações computacionais visando confirmar a reprodutibilidade do sistema pelo modelo. Detalhes da validação do modelo pode ser encontrada no trabalho de Silva et al. (2015).

Figura 2 - Comparação dos dados experimentais do trabalho de Silva et al. (2015) e asimulação das perdas de ácido ascórbico em função do tempo de reação.

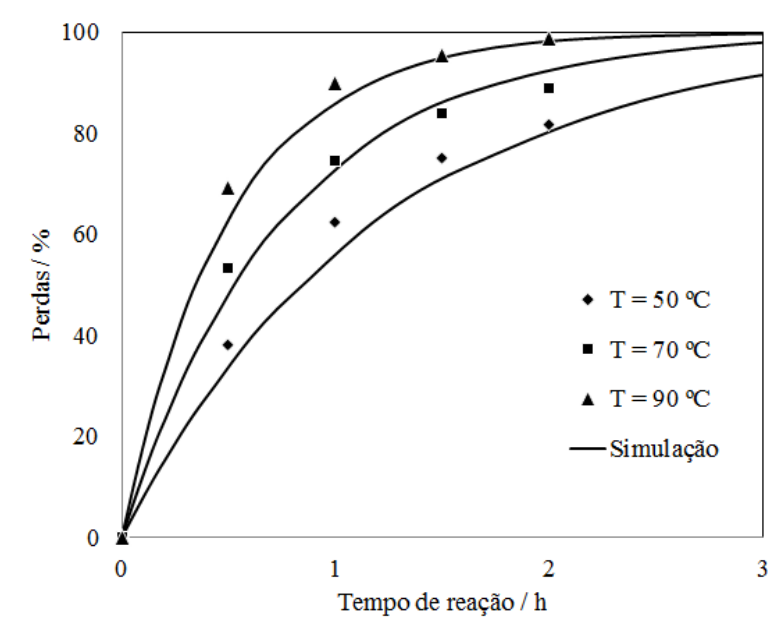

A Figura 2 claramente ilustra o efeito da temperatura no sistema. No entanto, é importante ressaltar que os processos de pasteurização normalmente possuem um tempo de residência de 5 a $20 \mathrm{~s}$ (Al-Zubaidy et al., 2006). Deste modo, aparentemente, para as três temperaturas analisadas, não haverá uma grande variação na degradação de AA no início do processo. Para confirmar este fato, a Tabela 2 foi construida para fornecer uma comparação mais detalhada do processo em um curto tempo de residência no reator tubular.

Tabela 2 - Simulação das perdas de AA no sistema tubular com tempo de residência de $20 \mathrm{~s}$ para três temperaturas distintas.

\begin{tabular}{cc}
\hline Temperatura $\left({ }^{\circ} \mathrm{C}\right)$ & Perdas simuladas $(\%)$ \\
\hline 50 & 14,42 \\
70 & 14,67 \\
90 & 14,94 \\
\hline
\end{tabular}

Com base nos resultados das simulações expressos na Tabela 2, percebe-se que para um pequeno tempo de residência do sistema, as perdas de AA são aproximadamente iguais. 
Neste caso, a temperatura do sistema não apresenta uma grande influência na degradação. Este resultado pode ser atribuído ao baixo valor da energia de ativação para o sistema $(21 \mathrm{~kJ}$ mol $^{-1}$ ) determinado no trabalho de Silva et al. (2015). Sabe-se que a degradação de ácido ascórbico em sucos cítricos pode variar de 20 a $53 \mathrm{~kJ} \mathrm{~mol}^{-1}$ (Vieira et al., 2000) e, quanto menor o valor da energia de ativação, menor será a influência da temperatura sobre a constante de degradação. Consequentemente, este fato terá uma baixa influência sobre a taxa de degradação do composto. Logo, o tempo de residência terá o papel crucial na degradação de AA para o sistema considerado. A Figura 3 fornece uma comparação da degradação do ácido ascórbico em três tempos de residência distintos, considerando uma temperatura de pasteurização igual a $70^{\circ} \mathrm{C}$.

Figura 3 - Simulação das perdas de AA em função do volume do reator num sistema a $70{ }^{\circ} \mathrm{C}$ considerando três tempos de residências distintos (1, 10 e $20 \mathrm{~s})$.

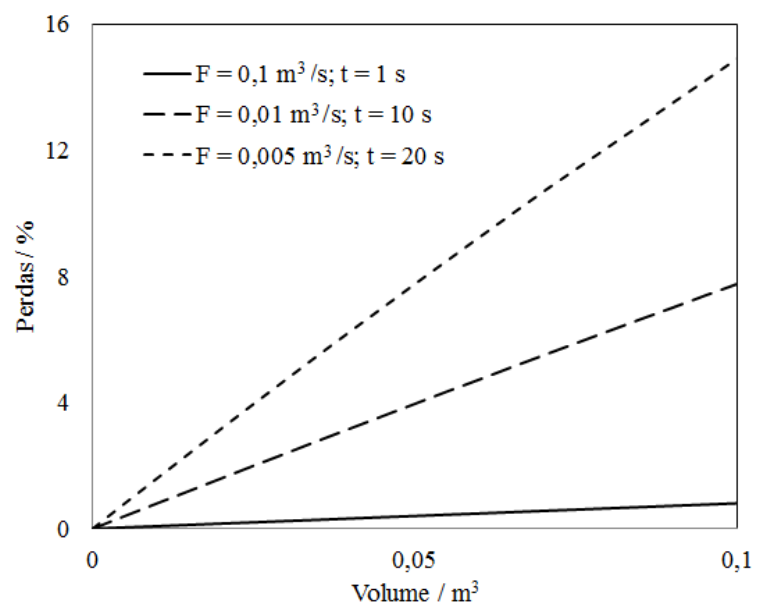

A Figura 3 comprara tempos de residência distintos no reator para uma mesma temperatura e, a partir dela, percebe-se que o parâmetro analisado exerce uma influencia bem maior do que a própria temperatura do sistema. Deste modo, os sistemas de pasteurização industriais devem operar com uma vazão tal que a inativação de enzimas seja eficiente, com a mínima degradação do ácido ascórbico. Em geral, os processos de pasteurização de sucos de laranja ocorrem numa temperatura em toro $80-90{ }^{\circ} \mathrm{C}$ e um tempo de residência menor do que $15 \mathrm{~s}$ (Zerdin et al., 2003). No estudo de caso do presente trabalho, sugere-se operar na maior temperatura $90{ }^{\circ} \mathrm{C}$, porém num tempo de residência de $10 \mathrm{~s}$. A maior temperatura forneceria uma maior eficiência na inativação de enzimas e microrganismos (Moya e Coichev, 2007). Em contrapartida, o menor tempo de residência faria com que as perdas de AA não ultrapassassem $10 \%$. É importante ressaltar que esses resultados são apenas um estudo prévio e, um estudo mais detalhado envolvendo cinética enzimática e microbiana seria necessária para obter as condições ótimas de operação para o sistema. Como trabalhos futuros, pretende-se explorar essas questões através de simulações em sistemas tubulares e de placas, visto que, até o momento nenhum trabalho foi encontrado na literatura trazendo essa abordagem.

\section{CONCLUSÃO}


O objetivo deste trabalho foi análise do tempo de residência e temperatura sobre a degradação de AA em suco de laranja "pêra" utilizado simulações. Verificou-se que as perdas de AA aumentaram de $5 \%$ para $14 \%$ no momento em que o tempo de residência foi reduzido pela metade em um sistema operando na mesma temperatura. Entretanto, quando um mesmo tempo de residência foi considerado $(20 \mathrm{~s})$, perdas similares de ácido ascórbico foram obtidas para três temperaturas distintas. Portanto, apesar de uma grande influência da temperatura sobre a degradação, o tempo de residência do suco no sistema de troca térmica é o fator que contribui de forma mais significativa no processo de pasteurização.

\section{AGRADECIMENTOS}

Os autores agradecem ao Instituto Federal do Sul de Minas Gerais, Campus Pouso Alegre - IFSULDEMINAS, pelo transporte fornecido à participação no evento.

\section{REFERÊNCIAS}

AL-ZUBAIDY, M. M. I.; KHALIL, R. A. Kinetic and prediction studies of ascorbic acid degradation in normal and concentrate local lemon juice during storage. Food Chem., v. 101, p. 254-259, 2007.

BIANCHI, M. L. P; ANTUNES, L. M. G. Radicais livres e os principais antioxidantes da dieta. Rev. Nutr., v. 12, p. 123-130, ago. 1999

BURDURLU, H. S; KOCA, N.; KARADENIZ, F. Degradation of Vitamin C in citrus juice concentrates during storage. J. Food Eng., v. 74, p. 211-216, 2006.

MANELA-AZULAYI, M. et al. Vitamina C. An. Bras. Dermatol, Rio de Janeiro, v. 78, n. 3, p. $1-10,2003$.

MOYA, D. H.; COICHEV, N. Kinetic studies of the oxidation of L-ascorbic acid by tris(oxalate) cobalt in the presence of CDTA metal ion complexes. J. Braz. Chem. Soc., v. 17, p. 264-368, 2006.

NEVES, E. M. et al. Citricultura brasileira: efeitos econômicos, Rev. Bras. Frutic., v. 23, n. 2, p. 432-436, 2001.

SILVA, N. L.; CRISPIM, J. M. S.; VIEIRA, R. P. Modelagem cinética da degradação térmica do ácido ascórbico em reator batelada. In.: XII Congresso Brasileiro de Engenharia Química em Iniciação Científica, Campinas, 2015.

VIEIRA, M. C.; TEIXEIRA, A. A.; SILVA, C. L. M. Mathematical modeling of the thermal degradation kinetics of vitamin $\mathrm{C}$ in cupuaçu (Theobroma grandiflorum) nectar. J. Food Eng., v. 43, p. 1-7, 2000.

ZERDIN, K.; ROONEY, M. L.; VERMUE, J. The vitamin C content of orange juice packed in an oxygen scavenger material. Food Chem., v. 82, p. 387-395, 2003. 